\title{
¿Qué posibilidad de discusión y acuerdo hay para Kant respecto de diversos juicios de gusto?
}

\author{
ANTONIO POMPOSINI \\ Pontificia Universidad Católica del Perú \\ https://doi.org/I0.18800/estudiosdefilosofia.20170I.003
}

Resumen: Este trabajo investiga la importancia que tiene la discusión sobre juicios de gusto dentro de la Crítica de la facultad de juzgar de Kant. Se sostiene que buscar acuerdos de facto sobre juicios de gusto es un error y que, más bien, el desacuerdo es deseable. Siguiendo a Kant en la antinomia del gusto, los juicios de gusto no se basan en algún concepto determinado, sino en uno indeterminado. Al no ser determinado, no tenemos intuición sensible de dicho concepto ni podemos emplearlo directamente. No se trata de encontrar un acuerdo de facto para nuestra aspiración de acuerdo universal, sino del descubrimiento de un sentido común, en el que encontramos que los otros pueden sentir como uno ante una representación dada. Este concepto indeterminado se torna en un "ideal regulativo", inalcanzable, en tanto no se puede determinar, pero al cual se debe apuntar para perfeccionar el gusto. Es en el campo de la discusión y el desacuerdo donde se confrontan los juicios de gusto.

Palabras Clave: antinomia, gusto, juicio, ideal, Kant.

Abstract: "Possibilities of Discussion and Agreement Regarding Kant's varied Judgments of Taste". This paper examines the importance that judgements of taste have within Kant's Critique of Judgement. It claims that looking for de facto agreements about judgements of taste is a mistake and that disagreements, instead, are desirable. Following Kant in the antinomy of taste, judgments of taste are not based on determined concepts, but rather on an undetermined one. Since the concept is not determined one cannot exhibit it in a sensible intuition or make direct reference to it. It is argued that universal agreement does not seek de facto agreement, but rather the discovery of a common sense in which one discovers that others can feel the same as one does with respect to a given representation. This undetermined concept becomes a "regulative ideal", unobtainable in so far as one cannot determine it, but to which one must aim 
in order to perfect one's taste. It is in the sphere of discussion and disagreement that one confronts one's judgements of taste.

Keywords: antinomy, taste, judgement, ideal, Kant.

\section{$\S 1$ 1. Introducción}

El presente trabajo tiene como objetivo central examinar la importancia que tiene discutir sobre juicios de gusto dentro de la Crítica de la facultad de juzgar de Kant. El problema se encuentra principalmente en la antinomia del gusto, al inicio de la Dialéctica de la facultad de juzgar estética y, a grandes rasgos, consiste en lo siguiente: los juicios de gusto no son meramente subjetivos (y privados) puesto que tienen pretensión de universalidad (aspiran a la aceptación de cada cual). Sin embargo, y al mismo tiempo, esta pretensión jamás puede concretarse definitivamente puesto que el juicio de gusto no se determina por argumentos probatorios o conceptos objetivos; es imposible probar que un juicio cualquiera esté en lo correcto. Siendo este el caso, ¿qué sentido tiene entablar discusiones sobre el juicio de gusto? El juicio de gusto parece estar destinado a permanecer incompleto por la simple imposibilidad de concretar su demanda. Sin embargo, este ensayo encuentra que el acuerdo no es la finalidad del juicio, sino más bien todo lo contrario; el desacuerdo es deseable e incluso preferible porque este permite reevaluar el juicio, preguntándose en qué puede estar equivocado, para así reorientarlo más acertadamente hacía la corrección. En esta perspectiva, el acuerdo nunca se realiza de manera concreta, sino que se mantiene como un "ideal regulativo" (Früchtl 1994) hacia el que todos apuntamos para refinar nuestros juicios, pero que nunca podemos verdaderamente obtener ( $y$ es preferible que no lo obtengamos, porque en dicho caso perderíamos la posibilidad de aproximarnos a la corrección del juicio y nos quedaríamos en un callejón sin salida). En esta interpretación, el rol de la discusión es crucial, pues es la herramienta principal a través de la cual confrontamos nuestros juicios de gusto, pedimos que el otro concuerde con nosotros, reconocemos las diferencias y emprendemos un rol crítico sobre nuestro propio juicio y el de los demás para aproximarnos cada vez más a un ideal regulativo de belleza. 
Se estudiará el rol central de la discusión en los juicios de gusto en dos pasos y secciones. La primera sección se dedicará a exponer el problema de la antinomia del gusto, indagando en la posibilidad de discutir sobre juicios de gusto y sobre la posibilidad de un fundamento objetivo de este. La antinomia del gusto se sostiene por la imposibilidad de basar juicios de gusto en conceptos determinados, mientras que, a la vez, este está basado en un concepto indeterminado, inalcanzable, pero con realidad objetiva. Este concepto indeterminado se convierte en el ideal de acuerdo al que todos los hombres buscan llegar refinando su juicio. Luego se explicará brevemente la importancia de la apelación al concepto indeterminado y el lugar que ocupa en la filosofía de Kant, así como las discusiones al respecto. La segunda sección retomará el problema de la discusión de los juicios de gusto para preguntarse si la imposibilidad del acuerdo es perjudicial o más bien beneficiosa. Se concluye que la discusión permite refinar los juicios de gusto y que además es una herramienta crucial para crear comunidad y colaboración, puesto que, a través de la posibilidad de sentir de la misma forma, o de un sentir común, este ideal regulativo es una colaboración comunitaria para crear espacios de concordancia.

\section{$\S 2$. La antinomia del gusto}

Para poder discutir propiamente los problemas que se presentan en la antinomia del gusto es imprescindible primero saber en qué consiste un juicio de gusto para Kant. Es en la Analítica de lo bello donde Kant se plantea realizar esta descomposición de los elementos de un juicio de gusto para encontrar en qué se fundamenta y qué es lo que pretende. Este trabajo no se propone ser una discusión sobre el desarrollo de la analítica; únicamente nos remitiremos a ella para esclarecer qué entiende Kant por un juicio de gusto.

Antes que nada, Kant define al juicio de gusto como un juicio estético. Esto significa, para él, que “(...) su fundamento de determinación no puede ser de otro modo sino subjetivo (...)” (Kant I99I, §I, B4). Con esto, distingue claramente los juicios de gusto de un juicio lógico de conocimiento. Mientras este último concierne al polo objetivo de una representación, el primero concierne al sentimiento de placer y displacer, que no es algo propio del objeto sino más bien del sujeto que es afectado por una representación. Aun así, el juicio de gusto no es meramente subjetivo, sino que tiene una pretensión de validez 
universal. A pesar de que el juicio no se determina por conceptos del entendimiento, no se concede que cada uno tenga su propio gusto (como ocurre con lo meramente agradable), sino que "(...) se le atribuye la complacencia de un objeto a cada cual, sin fundarse, no obstante, en un concepto (pues entonces sería lo bueno) (...)” (Kant 199I, §8, B22). Esto deja al juicio de gusto en una posición peculiar que Kant detalla en la deducción. En § 32, determina que un juicio de gusto apela a la aprobación de cada uno, como si fuera objetivo. En § 33, señala que "El juicio de gusto no es determinable por argumentos probatorios, tal como si fuese meramente subjetivo (...)” (Kant 199I, §33, BI40). En otras palabras, el juicio de gusto no es meramente subjetivo, ya que apela a la aprobación universal de cada cual, pero no puede ser meramente objetivo ya que no es determinable por argumentos probatorios (y no está basado en conceptos).

Teniendo en cuenta la naturaleza del juicio de gusto, preguntémonos: ipodemos concordar sobre nuestros juicios de gusto? Si no es posible resolver discusiones por medio de argumentos probatorios, no hay lugar para el acuerdo entre dos juicios de gusto distintos. Pero, si no se considerase siquiera la posibilidad de, en teoría, llegar a un acuerdo, ¿qué razón habría para discutir sobre juicios de gusto? Este es el problema que Kant plantea al inicio de la Dialéctica de la facultad de juzgar estética, en la antinomia del gusto. Una antinomia (y en particular la del gusto), como explica Allison, es “(...) un conflicto entre dos proposiciones igualmente necesarias, pero en apariencia contradictorias, que se siguen de principios generalmente aceptados del juicio estético (...)" (200I, 237, traducción nuestra). Wenzel (2005, I20-12I) agrega que una antinomia surge cuando razonamos más allá de nuestra capacidad al apelar a fundamentos a priori y que, por ello, es el rol de la dialéctica mostrar que estos principios no constituyen realmente una contradicción. Kant encuentra dos "lugares comunes" que sirven como principios generalmente aceptados del juicio de gusto: I) cada cual tiene su propio gusto y 2) sobre el juicio de gusto no se puede disputar. El primero implica que el juicio de gusto es meramente subjetivo, el segundo, que aunque el juicio pueda ser objetivo, no se define por conceptos determinados (Kant 199I, §56, B232-233). Estos dos lugares comunes no son contradictorios por sí mismos, sino, señala Kant, porque hay una proposición que se oculta entre las previamente expuestas: "Fácilmente se ve que entre estos dos lugares comunes falta una proposición que no circula proverbialmente, pero sí está contenida en el sentido de todos, a saber: 
Sobre gusto se puede discutir (aunque no disputar). Esta sentencia, empero, contiene lo contrario de la primera. Pues acerca de aquello sobre lo cual debe estar permitido discutir, tiene que haber la esperanza de llegar a convenir mutuamente; por consiguiente tiene que poder contarse con fundamentos del juicio que no tienen sólo validez privada y que, por tanto, no son meramente subjetivos; de lo cual es precisamente lo contrario aquel principio: cada cual tiene su propio gusto" (Kant 199I, §56, B233).

Kant señala explícitamente la diferencia entre dos tipos de desacuerdo: discutir y disputar. Ambos buscan llegar a unanimidad en los juicios, pero el segundo se diferencia del primero en tanto que este busca llegar a resolver el desacuerdo apelando a conceptos objetivos como fundamentos del juicio, mientras que el segundo permite sostener perspectivas opuestas, aunque sin la pretensión de resolver el desacuerdo mediante pruebas. Según lo previamente visto, es claro entonces que no se puede disputar sobre juicios de gusto, pues estos no se determinan por conceptos objetivos, pero vemos que Kant considera que, en tanto haya la esperanza de llegar a convenir mutuamente, sí es posible discutir sobre juicios de gusto. Pero esto lleva a un problema, pues para que haya esperanza de convenir mutuamente, el juicio de gusto no puede ser meramente subjetivo, y entonces encontramos una contradicción entre el primer lugar común y la proposición que afirma la posibilidad de discutir sobre juicios de gusto. Kant plantea esta contradicción en forma de una tesis y antítesis que constituyen la antinomia del gusto. La tesis sostiene que "El juicio de gusto no se funda en conceptos, pues de otro modo se podría disputar acerca de él (decidir a través de pruebas) (...)” (Kant I99I, §56, B234). La antítesis sostiene que "El juicio de gusto se funda en conceptos, pues de otro modo, sin atender a su diversidad, ni siquiera se podría discutir acerca suyo (apelar al necesario acuerdo de otros con este juicio) (...)” (Kant 199I, §56, B234).

Kant expone la solución a la antinomia en § 57. En la exposición de la solución, Kant inicia notando una similitud entre la tesis y la antítesis con las peculiaridades del gusto expuestas en la Deducción (§§32-33). En efecto, la tesis "el juicio de gusto no se funda en conceptos" puede emparejarse con la segunda peculiaridad del juicio, a saber, "el juicio de gusto no es determinable por argumentos probatorios, tal como si fuese meramente subjetivo". De igual manera, la antítesis "el juicio de gusto se funda en conceptos" es acorde con la primera peculiaridad del juicio, a saber, "el juicio de gusto apela a la aprobación 
de cada cual, como si fuese objetivo". Esta equiparación de la antinomia del gusto con las peculiaridades es motivo de debate entre quienes han trabajado el tema, ya que, como plantea Wenzel (2005, I2I), si el problema de las peculiaridades fue resuelto en la Deducción (\$38), entonces iqué necesidad habría de una dialéctica que reincide sobre el mismo problema? Dentro de la literatura sobre la Crítica del juicio se pueden dividir las posturas respecto de esta cuestión en dos grupos. El primero lo forman intérpretes que sostienen que la Dialéctica es en realidad una compleción de la Deducción, pues esta es insuficiente para legitimar la pretensión de los juicios de gusto y solo se concreta a través de la Dialéctica. En este grupo se encuentran autores como Crawford (1974), Brandt (1989), Elliot y Guyer (Rind 2000, 67). El segundo agrupa a quienes sostienen que la Deducción está completa en sí misma y que la Dialéctica tiene una tarea distinta; en este grupo podemos colocar las interpretaciones de Allison (200I) y Wenzel (2005).

Consideremos el primer grupo. Crawford sostiene que "The complete deduction of judgements of taste, the justification of our right to make judgements of the form 'This is beautiful' that demand the assent of others, is an extended and complicated argument occupying the entire first part of the critique of judgement, the 'Critique of Aesthetic Judgement' (...)" (Crawford 1974, 66). Es decir, Crawford plantea que la Deducción trascendental de los juicios de gusto no se concreta en $\S 38$, sino que está presente a lo largo de toda la primera parte del libro. En particular, cita a §§ 7-8 (en el segundo momento de la Analítica), §§ 32-33 (las peculiaridades que anticipan la Deducción) y §§ 56-57 (la antinomia del gusto) (Crawford 1974, 63). Brandt sostiene una postura similar, pero enfatiza que la necesidad que postula el juicio de gusto es una necesidad práctica; es decir, que el acuerdo debe darse de facto: "If necessity is to be derived from universality, the deduction has not achieved the goal of justifying the practical necessity of everyone else agreeing with my feeling and judgement of taste which Kant has asserted (...)" (Brandt 1989, 180). Así, sostiene que la Deducción no ha cumplido el objetivo de justificar la necesidad del acuerdo de todos y la vincula, más bien, con la Dialéctica: "In any case, the complex meant by 'our deduction' is not identical with the deduction in the 'Analytic' but rather refers to the proof in the context of the resolution of the antinomy of taste (...)" (Brandt 1989, 180). Más específicamente: “In the following I want to maintain the thesis that in fact the concept of the beautiful and with it the pure judgement of taste is only completely deduced 
in the 'Dialectic'. The claim which the judgement of taste makes for general approval cannot be upheld without recourse to the idea of the supersensible which is first available to the pure judgement of taste in the 'Dialectic'. Is this thesis correct, and if so, how can the dialectic, the logic of illusion, have the positive function by which it becomes in a certain sense the sphere of truth and not of deception and of illusion?" (Brandt 1989, I8I-182).

Con esta cita queda absolutamente claro que Brandt considera a la Dialéctica como la compleción del argumento de la Deducción. Sin embargo, la cita nos señala algo más: Brandt está pretendiendo que la Dialéctica tenga una función positiva o constructiva, pero esto es distinto a la concepción kantiana de dialéctica que, como explica Wenzel, tiene siempre la función negativa de disolver las contradicciones aparentes a las que llegamos cuando pretendemos llegar más allá de nuestros límites a través del uso de la razón (2005, 120). En oposición a lo previamente expuesto, Wenzel sostiene que la Dialéctica concierne a un problema distinto del que se trata en la Deducción y que la antinomia no retoma el problema de las peculiaridades del gusto, sino que, aunque semejantes en formulación, tienen objetivos completamente distintos, pues no tendría caso que Kant realice una dialéctica si el problema a tratar ya ha sido resuelto previamente en la obra: "I suggest that the thesis and antithesis, which create the antinomy (in the Dialectic), should be understood as generalizations, or inflations, of the two earlier peculiarities. The thesis and antithesis are talking about the judgment of taste and about its general nature and principle. The contradiction of the antinomy should be seen as one of those principles and justifying grounds, those 'concepts' that are mentioned in the thesis and antithesis. On the other hand, the contradiction that one finds in the judgment of taste and its peculiarities (in the Analytic) should be seen more on the level of the judgment of taste itself, for instance in the case where my judgment about this rose goes against yours, where I find the rose beautiful whereas you do not (and where I demand that you and everyone else should agree to my judgment). That is, I suggest, the antinomy takes place on a different level (the level of principles)" (Wenzel 2005, I2I-I22).

Esta cita hace eco del § 55, en el que Kant explica que no puede haber una dialéctica sobre los juicios estéticos (tanto de lo agradable como de gusto). Incluso dice: "No resta, pues, ningún concepto de una dialéctica que pudiera concernir al gusto más que el de una dialéctica de la crítica del gusto (no del 
gusto mismo) en vista de sus principios (...)” (Kant I99I, §55, B232). La antinomia, para Wenzel, está constituida por principios que son generalizaciones de las peculiaridades del gusto expuestas antes, pero no están al mismo nivel que las peculiaridades. Allison entiende el problema en la misma línea que Wenzel, diciendo que: "I take Kant's point not to be that the thesis and antithesis are each to be simply equated with one of the two peculiarities, but rather that the former are derived from the latter by a process of absolutization. In other words, the antinomy is generated through the inflation of what is initially merely a moment or aspect of the judgment of taste into a free-standing principle that expresses the whole truth regarding taste" (Allison 200I, 242). En otras palabras, esta absolutización elimina los como si presentes en las peculiaridades. La antítesis no dice que discutimos sobre el juicio como si fuese objetivo, sino afirma que el juicio se funda en conceptos (hay objetividad en juego) (Allison 200I, 242). Esta absolutización distingue las proposiciones de la Antinomia de las peculiaridades.

Entonces se ve que en la Antinomia la preocupación no puede ser la misma que en la Deducción, puesto que las proposiciones no son "extensiones" de las peculiaridades, sino que, aunque relacionadas, se dirigen a un problema distinto; en este caso, la tarea negativa de mostrar que no hay contradicción a nivel de la razón sobre los principios del juicio de gusto. Así, Kant sería consecuente con la pauta establecida en las otras dos Críticas. Ahora bien, ¿cómo se soluciona la aparente contradicción presente en la Antinomia del gusto? Se resuelve por medio de una especificación del término "concepto": "El juicio de gusto tiene que referirse a un concepto, pues de otra manera no podría en absoluto tener pretensión de validez necesaria para todos (...)" (Kant 199I, §57, B234-235). Pero no pueden ser conceptos determinados (del entendimiento), pues estos servirían de prueba para el juicio de gusto. Por ello, Kant sostiene que el concepto al que se refiere la antítesis no es un concepto determinado, sino uno indeterminado (e indeterminable): el "concepto trascendental de razón de lo suprasensible” (Kant 1991, §57, B235). Solo es posible disponer de un concepto indeterminado apelando a la razón ya que, como dice Allison, “(...) since to determine a concept is to provide it with a corresponding sensible intuition, this amounts to a distinction between concepts that can be provided with such an intuition and those that cannot (...)" (200I, 245). Además, sabemos que Kant afirma que, aunque es imposible tener una intuición sensible de algo noumenal (como lo absolutamente grande 
por ejemplo), es perfectamente posible tener una intuición sensible para los conceptos del entendimiento. Así pues, una vez aclarada la ambigüedad respecto del término "concepto" (hay conceptos del entendimiento e ideas de la razón), Kant reformula la Antinomia de la siguiente manera: "En la tesis debería (...) decir: el juicio de gusto no se funda en conceptos determinados; en la antítesis, en cambio: el juicio de gusto se funda en un concepto, aunque indeterminado (a saber, del substrato suprasensible de los fenómenos) (...)" (1991, §57, B237).

La apelación al substrato suprasensible es otro asunto en el que hay que detenerse ( $y$ en el que muchos intérpretes se han detenido). Kant dice que no hay mayor discusión posible respecto de lo suprasensible y que basta con apelar a este para disolver la contradicción de la Antinomia. En efecto, ya que Kant apela a un concepto de la razón, este está más allá de nuestra capacidad de conocerlo y, por ello, de determinarlo. Esto, empero, no impide que hayan consideraciones relevantes al respecto. La apelación a lo suprasensible no debe concebirse como una salida arbitraria, deus ex machina, al problema planteado por la Antinomia, sino que proviene y forma parte del sistema de la filosofía trascendental. Los intérpretes discuten sobre cuál puede ser el concepto de lo suprasensible al que apela Kant. Así, Wenzel $(2005,123)$ lo identifica con el principio de la conformidad a fin sin fin (o subjetiva), Allison (200I, 244) explícitamente entiende a lo suprasensible como el concepto de lo bello y Guyer (Allison 200I, 246-247) lo identifica, más bien, con la armonía de las facultades de conocimiento. Consideremos más en detalle sus posiciones.

Wenzel argumenta que lo suprasensible debe cumplir con dos criterios: “(I) It must establish the judgment of taste as an a priori judgment, so that an antinomy of principles (not just an empirical contradiction between particular judgments of taste) can arise; and (2) it must allow for a solution to this antinomy. This solution then helps (...) to show that reason is without contradiction, or, rather, that our philosophical concept of it is without contradiction" (2005, 123). Encuentra que la conformidad a fin sin fin es un candidato ideal para cumplir con ambos criterios, ya que tiene una base a priori (criterio I) y es indeterminable (criterio 2). Más aun, Wenzel propone que el concepto de lo suprasensible es crucial para la pretensión de Kant de sobrepasar la distinción entre la naturaleza y la libertad y, así, unificar el proyecto crítico. Por ello se 62 dedica a explorar el lugar que tiene lo suprasensible a lo largo de la Crítica del 
juicio, enfatizando las secciones de la Crítica a la facultad de juzgar teleológica en las que Kant elabora más sobre la posibilidad de una perspectiva distinta a la del entendimiento, pero acorde con la razón, capaz de captar a la naturaleza con un "entendimiento intuitivo" (2005, 123-125)'.

Por otro lado, Guyer cree que tal apelación solo está para servir, injustificadamente, al proyecto trascendental de Kant, y que existe otro candidato como concepto indeterminado, además de la idea de lo suprasensible, que funciona de igual forma sin tratar de justificar un idealismo, a saber, el concepto de la armonía o libre juego de las facultades de conocimiento (Allison 200I, 246). Sin embargo, en respuesta a la propuesta de Guyer, Alison explica: "For present purposes, however, the main point is that an indeterminable concept is an idea of reason and that such a concept is required for the resolution of the antinomy, since a merely indeterminate (but determinable) concept would hold open the possibility of proof (through its determination). Otherwise expressed, only such a 'concept' could be viewed as having the normative standing required to ground the possibility of genuine disagreement, while at the same time precluding the possibility of the determination needed to provide a rule capable of yielding a decision procedure for disputes about taste" (Allison 200I, 248).

Es decir, el problema es que un concepto como la armonía de las facultades es determinable, al menos en principio, y ello llevaría a que se puedan determinar los juicios de gusto por argumentos, si tan solo se conociese empíricamente (quizás a través de una investigación psicológica como sugiere Guyer) la proporción objetiva de las facultades de conocimiento. Una idea de la razón, que es indeterminable, es, en cambio, la adecuada para servir como concepto indeterminado, ya que es imposible, siquiera en principio, que podamos determinar por medios empíricos el concepto al que apunta un juicio de gusto. Esto, nuevamente, nos deja en una posición curiosa, en la que discutimos sobre juicios de gusto (y estamos legitimados a hacerlo, ya que existe un concepto indeterminado al que nos referimos) pero, por la propia naturaleza del juicio, es absolutamente imposible, siquiera en principio, llegar

I Señalemos, brevemente, que Weiler (1962) estudia precisamente el concepto de lo indeterminado y cómo es que conecta a la Crítica del juicio con la unificación del proyecto trascendental. 
a zanjar una discusión sobre juicios de gusto. ¿Qué importancia tiene discutir si jamás podemos probar que estamos en lo correcto al decir que algo es bello? Responderemos a esta cuestión en la siguiente sección.

\section{§ 3. ¿Qué lugar tiene la discusión en los juicios de gusto?}

Estamos ante un dilema: el juicio de gusto aspira a una aceptación universal, pero todo intento por demostrar la necesidad del acuerdo respecto de un juicio queda inevitablemente frustrado porque el juicio ha de ser conceptualmente indeterminado. Entonces, ¿qué propósito tiene la discusión sobre los juicios de gusto si no hay forma de garantizar el acuerdo? Cabe resaltar aquí que el acuerdo no es imposible; en efecto, bien podría haber dos o más personas que juzguen de la misma manera. Pero si dos personas juzgan distinto, ¿qué sentido tiene que discutan al respecto, si no hay posibilidad de que uno le muestre a otro que tiene la razón? En lo que sigue intentaremos mostrar porqué la discusión sobre juicios de gusto, a pesar de su aparente inutilidad, es altamente importante y que, además, es clave para entender propiamente la posición de Kant sobre los juicios estéticos.

Stern (1991, 69-70) considera que una posición como la de Kant, que admite la posibilidad de discutir sobre juicios de gusto, pero únicamente si nos sometemos a la creencia en un principio objetivo incognoscible, no es deseable porque niega la pluralidad de juicios y elimina la diversidad, rechazando la posibilidad de que haya diversos juicios de gusto igualmente válidos. Stern afirma que Kant le da demasiado valor a la convergencia, a costas de la diversidad, porque quiere “(...) preservar su convicción de que, en principio, existe una determinación objetiva del gusto, a pesar de su incapacidad para encontrar un buen argumento a favor (...)" (1991, 70, traducción nuestra). Desestimar la apelación a lo suprasensible porque es "desfavorable" no es motivo para hacerlo pero, más allá de ello, el lugar de la convergencia en Kant no es meramente un pretexto para mantener sus concepciones metafísicas, sino que es un elemento crucial del juicio estético que no se debe desestimar en absoluto si se quiere elucidar el potencial significado de un juicio tal. $\mathrm{Si}$ no hubiera posibilidad de convergir y cada uno tuviese su propio gusto, no habría siquiera necesidad de discutir sobre estética, pero si todos pudiesen estar de acuerdo, tampoco habría discusión, pues todos emplearían el mismo 
argumento. Por ello, el hecho de que haya discusión es una posición mucho más fructífera, ya que nos permite percatarnos de una peculiaridad sobre cómo juzgamos los seres humanos.

Es importante recalcar que Kant no niega, en ningún momento, la diversidad de los juicios de gusto. Muy por el contrario, es consciente de que la gente frecuentemente entra en desacuerdos respecto de juicios de gusto. "El juicio de gusto exige de cada cual asentimiento; y quien declara a algo bello quiere que cada uno deba dar su aprobación al objeto allí presente y llamarlo igualmente bello (...)" (Kant 199I, § 19, B 63). El juicio de gusto no requiere una necesidad práctica, o de facto, para ser legitima (como insistiría Brandt), sino que basta con tan solo postular, y aspirar a, una necesidad ideal. Lo peculiar del juicio de gusto es que nunca puede forzarse a que ocurra de cierta manera y no puede determinarse por reglas generales; la necesidad del juicio de gusto es “(...) ejemplar, es decir, [se trata de] la necesidad del asentimiento de todos a un juicio que es considerado como ejemplo de una regla universal que no puede ser aducida" (Kant 199।, §18, B62-63). En otras palabras, un sujeto que juzga jamás puede estar seguro de que el caso en cuestión se subsume correctamente a la regla, pero, ya que el juicio no está basado en conceptos, la única referencia que se tiene sobre el juicio es el juicio mismo. En § 19, Kant dice (a nuestro entender) que si se pudiese estar seguros de haber subsumido correctamente la regla, contaríamos de facto la aprobación de todos (que también habrían subsumido correctamente), ya que el fundamento del juicio es común a todos los que juzgan. Este fundamento común, como explica Kant en $\S \S 21-22$, es un sentido común; es decir, la capacidad de cada cual de sentir de la misma forma que otros.

Como se ve en $\S \S 32-33$, las peculiaridades del gusto señalan que nadie puede convencer a otro de sentir de una determinada manera, ni porque ofrezca argumentos ni porque la mayoría convenga en la corrección de un juicio. El sujeto debe juzgar de forma autónoma y, a la vez, de manera válida para todos. Pero que se deba juzgar de manera autónoma no invalida la suposición de un sentido común a todos: "(...) what the subject finds in the moment when it turns inward to reflect on itself is not the essence of pure subjectivity, the I $=\mathrm{I}$ secured in the Cartesian cogito, but a universal voice, which transcends and traverses the subject, speaking itself through the subject in the aesthetic judgment (...)" (Soni 2006, 8). Es decir, el sujeto, al reflexionar sobre su sentir, 
se ve impulsado a determinar el juicio por la vía reflexionante, ignorando los argumentos y el juicio de los demás. Sería un acto solipsista, de la más profunda subjetividad, si no fuese porque son sus facultades las que entablan una relación libre, susceptible de una "voz universal" o un sentido común. Es en virtud de tal relación (cuyo índice es el placer) que el sujeto puede pretender que otros puedan sentir de la misma forma que él.

Ahora bien, aunque el sujeto deba juzgar de manera autónoma, el que esté en desacuerdo con la mayoría sería de todas formas motivo de sospecha: "EI juicio de otros que nos fuese desfavorable puede hacernos sospechar $y$, por cierto, con derecho, del nuestro, pero jamás nos convencerá de la incorrección del mismo (...)" (Kant 199I, §33, BI40-14I). Entonces, como expone Früchtl $(1994,61)$, hay dos criterios que son indicadores de haber subsumido la regla correctamente: I) el juicio debe ser válido para todos y 2) el juicio se determina de manera autónoma. Según Früchtl, "Los dos criterios cojean pues de una deficiencia complementaria. El primero sería suficiente pero no es verificable ni para uno ni para los demás; por ello no es un criterio. El segundo es verificable pero no suficiente (...)" $(1994,61)$.

El juicio de gusto tiene pretensión de universalidad, pero para lograr esta universalidad tenemos que haber subsumido correctamente el caso bajo la regla y juzgar desde el sentir común. No podemos jamás confirmar que hemos subsumido bajo la regla correctamente, pero asumimos que somos capaces de sentir en común (evidenciado por el hecho de que aspiramos a universalidad en nuestros juicios estéticos). Por esto es que la discusión respecto de juicios estéticos se torna de particular importancia. No podemos confirmar que hemos subsumido correctamente, solo tenemos indicadores: nuestro sentimiento de placer o displacer y el acuerdo o desacuerdo de otros. Si nadie está de acuerdo con mi juicio de gusto, eso es un indicador de que puedo haber subsumido incorrectamente y debo, entonces, reevaluar mi juicio. Si no encuentro bello un objeto que la mayoría juzga como tal, eso puede ser un indicador de que quienes así lo hicieron han errado en su juicio o, por el contrario, de que mi gusto no está lo suficientemente refinado o ejercitado como para captar el caso. Por esto Kant plantea máximas para tratar de aproximarse al sentir común: “I. Pensar por sí mismo; 2. Pensar en el lugar de cada uno de los otros; 3 . Pensar siempre acorde consigo mismo. La primera es la máxima del modo de pensar desprejuiciado. La segunda lo es del amplio 
y la tercera, del consecuente (...)" (Kant 199I, §40, BI58). La importancia de estas máximas para aproximarse al sentir común es inmediatamente evidente gracias a lo que sostiene Crawford: "In other words, by abstracting from our own limitations and the idiosyncrasies that contingently attach to our own judgment, we put ourselves in the place of any other person and base our judgments on what can be common to all men -the formal purposiveness of the way the object appears. Thus the principle of taste is just a common sense - the ability to limit one's powers of judgment to a feeling based on what is universally communicable -the form- and abstract from all other factors. In this way a common sense is postulated by the judgement of taste. Since this procedure of the power of judgment must also be exercised in all judgments, including cognitive judgments, we are justified in assuming a common sense" (Crawford 1974, 131).

Debemos tener una “(...) idea regulativa, (...) una suposición inevitable tanto como un ideal jamás realizable (...)” (Früchtl 1994, 55). Este ideal es la correcta subsunción del caso a la regla (o la conformidad a fin formal), algo que aspiramos lograr con nuestro juicio de gusto, pero que jamás podemos asegurarnos de haber obtenido. Debemos siempre estar abiertos a reevaluar nuestro juicio ya que nunca podemos cerciorarnos de su corrección, pues de esta manera siempre estaremos refinando nuestra capacidad de juzgar de una forma subjetiva-universal; es decir, de ponernos en el lugar de otros, a través de una determinación puramente subjetiva, y sentir como cualquiera puede sentir, porque podemos usar nuestras facultades de una misma manera, con un sentido común. Por esto es que la discusión es tan crucial para el juicio de gusto. Si nos encontráramos siempre de acuerdo, no habría necesidad de revisar nuestro juicio y, de esa forma, no podríamos reevaluar si (y qué) compartimos con otros; mientras que si asumimos un ideal regulativo al que siempre apuntamos, entonces podemos constantemente confrontar nuestros juicios con dicho ideal, buscando conseguir consensos, aunque nunca puedan ser totales. La imposibilidad del acuerdo es tan importante porque permite la discusión, y la discusión es la única forma que tenemos de aproximarnos a la correcta subsunción a esa regla común.

Lo curioso es que esta capacidad de sentir en comunidad, como Soni (2006) demuestra excelentemente, es una peculiaridad del ser humano: la de estar siempre abierto a formar comunidad y a crear consenso. El ser humano, 
entonces, tendría una disposición a buscar que otros estén de acuerdo con uno y a estar de acuerdo con otros; en otras palabras, a sentir en comunidad: "The unlikely performance of an aesthetic judgment leads us each time to an encounter with the remarkable possibility of a sensus communis, which breaches the solipsism of the subject, a common sense, which is also the sense of community. Aesthetic judgment discloses a structure that is essential and that nothing else within the subject can disclose: the possibility of and opening to community at the heart of subjectivity. The interest of aesthetic judgments, then, lies in the experience of community that they always intimate in the universal voice. The sensus communis is the raison d'être of the third critique" (Soni 2006, 8-9).

El ideal regulativo bajo el que nos regimos en el juicio estético para poder sentir el placer universal en la belleza es, entonces, solo un ejemplo de nuestra manera de proceder para sentir en comunidad, de nuestra apertura a sentir como los demás y a entendernos y comunicarnos. Como sostiene Soni (2006 9-10), la presuposición de sentir en comunidad es algo que antecede incluso a la comunicación racional y que es la base para toda comunicabilidad y comunión humana. En este sentido, la Crítica de la facultad de juzgar es una pieza clave para el sistema crítico de Kant, puesto que se convierte en la base que posibilita y articula las dos Críticas previas, unificándolas bajo el sentido común, la conformidad a fin y el ser humano.

\section{Bibliografía}

Allison, Henry, 200I. Kant's Theory of Taste. Nueva York: Cambridge University Press. https://doi.org//0.1017/CBO97805/161267|

Brandt, Reinhardt, 1989. Analytic/Dialectic. En: Reading Kant: New Perspectives on Transcendental Arguments and Critical Philosophy, eds. Eva Schaper y Wilhelm Vossenkuhn. Nueva York: Basil Blackwell, I79-195.

Crawford, Donald, 1974. Kant's Aesthetic Theory. Madison: University ofWisconsin Press. Früchtl, Josef, 1994. De la comunicabilidad de lo no comunicable. Una revisión de Kant. Analys-art II, 43-65.

Kant, Emmanuel, 1991. Crítica de la facultad de juzgar. Caracas: Monte Avila. 
Rind, Miles, 2000. What Is Claimed in a Kantian Judgement of Taste?. Journal of the History of philosophy 38 (I), 63-85. https://doi.org//0.1353/hph.2005.0103

Soni, Vivasvan, 2006. Communal Narcosis and Sublime Withdrawal: The Problem of Community in Kant's “Critique of Judgment”. Cultural Critique 64, I-39. https://doi. org/I0.I353/cul.2006.0030

Stern, David, 1991. Are Disagreements about Taste Possible? A Discussion of Kant's Antinomy of Taste. The lowa Review 21 (2), 66-7I.

Weiler, Gershon, 1962. Kant's “Indeterminate Concept” and the Concept of Man. Revue Internationale de Philosophie 16 (6I/62), 432-446.

Wenzel, Christian, 2005. An Introduction to Kant's Aesthetics: Core Concepts and Problems. Oxford: Blackwell. https://doi.org/I0.1002/9780470776599 